\title{
Recombination of charge carriers in heterostructures with Ge nanoislands grown on $\mathrm{Si}(100)$
}

\author{
S.V. Kondratenko \\ Taras Shevchenko Kyiv National University, \\ 64/13,Volodymyrs'ka str., 01601 Kyiv,Ukraine; e-mail: kondr@univ.kiev.ua
}

\begin{abstract}
In this paper, the study of the recombination of non-equilibrium charge carriers and determination of recombination mechanisms in $\mathrm{Ge} / \mathrm{Si}$ heterostructures with nanoislands have been presented. The effects of long-term photoconductivity decay in $\mathrm{Ge} / \mathrm{Si}$ heterostructures with Ge nanoislands have been found as caused by variations of the electrostatic potential in the near-surface region of $\mathrm{Si}(100)$ substrate and spatial separation of electron-hole pairs between localized states of Ge nanoislands and states of wetting layer and $\mathrm{Si}$. It has been shown that the photoconductivity decay depends on the excitation energy and temperature, while Ge nanoislands are Shockley-Read recombination centers with a higher recombination rate as compared with $\mathrm{Si}$.
\end{abstract}

Keywords: photoconductivity, recombination, nanoislands.

Manuscript received 06.10.14; revised version received 14.12.14; accepted for publication 19.02.15; published online 26.02.15.

\section{Introduction}

Low-dimensional Ge/Si heterostructures have attracted considerable research interest in recent years due to their significant potential to impact new electronic devices which are compatible with the available silicon technology. Interest in semiconductor heterostructures with nanoscale objects is due to the size quantization effects that lead to changes in the electronic spectrum. Optoelectronic devices based on $\mathrm{SiGe}$ dots grown on a $\mathrm{Si}$ substrate have been already proposed [3, 4]. In particular, the use of interband or intraband transitions involving localized states in the valence band of $\mathrm{SiGe}$ can increase the photoconversion efficiency in the near infrared range. The low-dimensional silicongermanium alloys have a wide range of applications, including quantum dot IR photodetectors, memory cells and spintronic devices. Widespread application of this system is the arrangement of SiGe quantum dots in the space-charge region of heterojunctions, Schottky diodes, $p$ - $n$ junctions or metal-oxide-semiconductor structures $[1-5,10]$.

It is known that $\mathrm{Ge} / \mathrm{Si}$ heterostructures are related to the type II heterostructures where the potential well exists only for one type of charge carrier - holes in the valence band of Ge nanoisland. The valence band offset value in these structures depends on the composition of the nanoislands and their environment, on the presence of mechanical strain and on the influence of quantum confinement effects. In heterostructures $\mathrm{Ge} / \mathrm{Si}$ with $\mathrm{SiGe}$ quantum dots, spatial separation of non-equilibrium charge carriers takes place - holes in the valence band states are captured by $\mathrm{SiGe}$, and electrons are accumulated in the potential well of Si surrounding. As a result, the SiGe quantum dots at low temperatures can accumulate positive charges. Under these conditions, the presence of $\mathrm{SiGe}$ nanoislands on the surface of $\mathrm{Si}$ substrate affects on redistribution of the charge density along the epitaxial layers, which in turn affects the processes of recombination of charge carriers. 
In this paper, we present the results of analyzing the processes of photogeneration and recombination of non-equilibrium charge carriers in heterostructures with self-assembled $\mathrm{Ge}$ nanoislands on $\mathrm{Si}(001)$ substrate grown using the molecular beam epitaxy (MBE) technique as well as photocurrent spectroscopy and photocurrent decay techniques.

\section{Experiment}

The Ge nanocluster structures were grown using a MBE technique on boron doped $\left(N_{a} \sim 10^{15} \mathrm{~cm}^{-3}\right) \mathrm{Si}(001)$ substrates with the resistivity of $7.5 \Omega \cdot \mathrm{cm}$ is the Stranski-Krastanov growth mode [12]. For deposition monitoring, reflection high-energy electron diffraction (RHEED) was employed. After oxide removal, a welldefined $\mathrm{Si}(001)$ surface was achieved by depositing a $\mathrm{Si}$ buffer layer onto the silicon wafer until a high-contrast $\mathrm{Si}(001) 2 \times 1$ electron diffraction pattern was visible. Subsequently, Ge was deposited at the deposition rate close to $\sim 0.05 \AA / \mathrm{s}$ and the substrate temperature $600{ }^{\circ} \mathrm{C}$ resulting in a strain driven formation of $\mathrm{Ge}$ nanoislands with the lateral extensions between 60 and $450 \mathrm{~nm}$ and height between 60 and $450 \mathrm{~nm}$. Topographic measurements of $\mathrm{Ge}-\mathrm{Si}$ heterostructure were performed simultaneously under normal ambient conditions using NTEGRA (NT-MDT) system equipped with an $15 \times 15 \mu \mathrm{m}$ closed-loop scanner and an $650 \mathrm{~nm}$ super luminescent diode for readout of the cantilever bending.

Ohmic contacts separated by $10 \mathrm{~mm}$ from each other were formed by annealing $\mathrm{Au}$ on the surface at $370{ }^{\circ} \mathrm{C}$ in $\mathrm{N}_{2}$ ambient, to provide that conductivity measurements can be performed for in-plane, lateral transport. The dark current and the photocurrent were measured over the temperature range of $80 \ldots 290 \mathrm{~K}$ using a current amplifier and standard detection of the direct current. The experimental current-voltage curves were linear within the range from 77 to $290 \mathrm{~K}$ at low applied voltages, less than $500 \mathrm{mV}$, and demonstrating ohmic behavior. Transient photoconductivity (PC) and spectral measurements were done using excitation from a 250-W lamp spectrally resolved through a monochromator. Photoconductivity spectra were measured between 0.6 to $1.8 \mathrm{eV}$ with normal incidence light excitation and a low electric field of $10 \mathrm{mV} / \mathrm{cm}$, which was applied along [001] direction.

\section{Results and discussion}

Measurements of infrared photoconductivity in Ge nanoislands / Si structures made it possible to evaluate their electronic spectrum. Fig. 1 shows the spectral dependences of in-plane photoconductivity of $\mathrm{Ge} / \mathrm{Si}$ structure with $\mathrm{SiGe}$ nanoislands measured at different temperatures. The PC spectrum shape for the structure with $\mathrm{SiGe}$ nanoislands indicates the small $\left(\sim 10^{3} \mathrm{~cm} / \mathrm{s}\right)$ velocity of surface recombination. The inplane photocurrent in the range $h v>\varepsilon_{\mathrm{Ge}, \mathrm{Si}}$ is mainly originated from band-to-band transitions in $c$-Si. For light excitations with photon energy below the band gap of Si $h v<\varepsilon_{\mathrm{Ge}, \mathrm{Si}}\left(\varepsilon_{\mathrm{Ge}, \mathrm{Si}}=1.17 \mathrm{eV}\right.$ at $\left.77 \mathrm{~K}\right)$, the electronic transitions from the valence band to conduction band of $\mathrm{SiGe}$ nanoislands give their main contribution to PC. However, generation of photocurrent within the range $0.8<h v<\varepsilon_{\mathrm{Ge}, \mathrm{Si}}$ for $\mathrm{Ge} / \mathrm{Si}$ is also possible due to transitions between tails of the density of states in the near-surface $c$-Si, the optical absorption spectra of which are described by the Urbach rule.

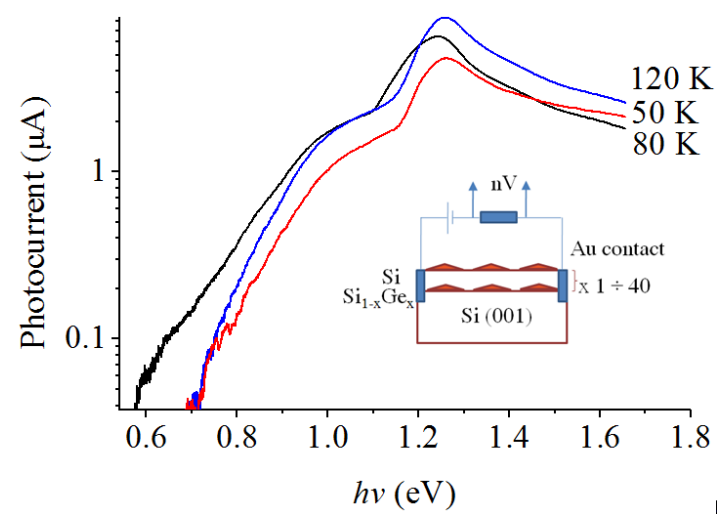

Fig. 1. In-plane PC spectra of the $\mathrm{Si} / \mathrm{Ge}$ heterostructure with nanoislands at different temperatures. The inset shows the setup for in-plane PC measurement.

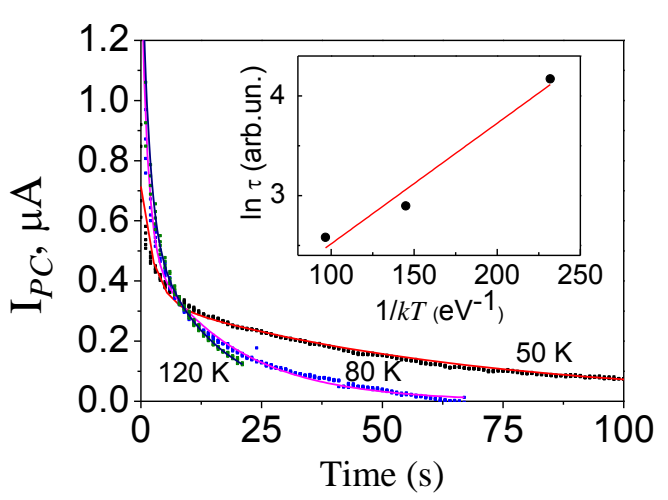

$a$

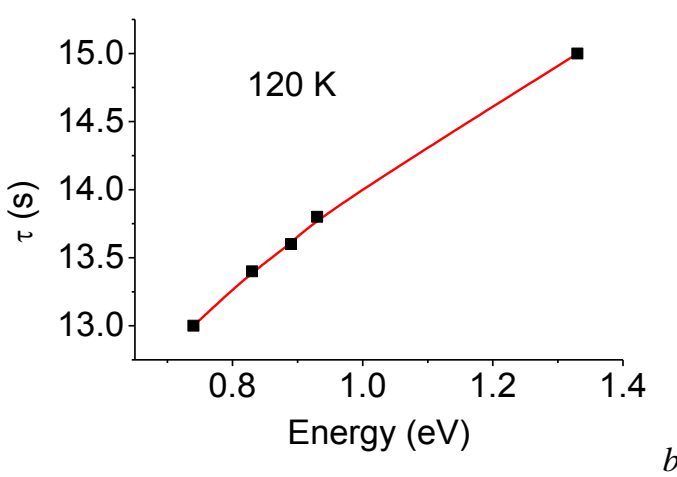

Fig. 2. (a) Time dependences of the photocurrent measured after photoexcitation by the quanta with the energy $h v=0.9 \mathrm{eV}$ at different temperatures: 50, 80 and $120 \mathrm{~K}$. (b) Spectral dependence of photocurrent decay constant of the sample at the temperature $T=120 \mathrm{~K}$. 
Fig. 2 shows the temporal evolution of photocurrent measured after the excitation by the quanta with the energy $h v=0.9 \mathrm{eV}$ at different temperatures: 50, 80 and $120 \mathrm{~K}$. After illumination, the current decays under the law:

$$
I=I_{0}+I_{\mathrm{PC}} \exp \left(-\frac{t}{\tau}\right),
$$

where $I_{0}$ and $I_{\mathrm{PC}}$ are the values of dark current and photocurrent, respectively, and $\tau$ is a decay constant. The activation energy $\varepsilon_{a}=12 \mathrm{meV}$ was extracted from the Arrhenius-type plot of $\ln \tau$ vs $1 /(k T)$, shown in the inset to Fig. 2a. The spectral dependence of the decay constant is shown in Fig. 2b. It was found that the decay constant increases with increasing the excitation quantum energy. The relatively faster relaxation after excitation of SiGe nanoislands, which is observed after $0.8-\mathrm{eV}$ excitation is the evidence of the fact that recombination involving the states of nanoislands is more effective in comparison with recombination in the wetting layer or Shockley-Read-Hall recombination centres in the depletion layer of Si substrate.

Several models were proposed to explain the origin of the observed long-term PC. In the microscopic localpotential model, the local fields of inhomogeneity separate photoexcited electrons and holes and thus delay recombination [8]. The other dominant mechanism involves macroscopic potential barriers, which prevent recombination in the other way $[9,10]$. One type of carriers should be localized by traps, while the other one are free and separated spatially. As reported in $[8,11]$, both the long decay and high conductivity values cannot be explained by usual models of Shockley-Read recombination centers. After optical illumination is removed, the long-term $\mathrm{PC}$ was observed due to recombination-preventing potential barrier, which separates spatially the regions with trapped carriers and the conductivity channel in $p$-Si substrate. The described macroscopic barrier originates from the space-charge region of near-surface $\mathrm{Si}$. In addition, availability of $\mathrm{Ge}$ nanoislands would induce in-plane variations of the electrostatic potential in the conductivity channel, which favours to spatial separation of photoexcited electronhole pairs in lateral direction. Large-scale electrostatic fluctuations could exist in the near-surface region of $\mathrm{Si}$ substrate, which are the result of spatial distribution of trapped electrons and holes, inhomogeneity of the wetting layer, interface imperfections, and non-uniform strains in Ge nanoislands and Si substrate. Moreover, the strain-modified confinement potential for electrons in underlying silicon can enhance in-plane fluctuations for Ge nanoislands / Si structures [12]. In the work [13], for example, the authors found that inhomogeneous deformation can cause significant changes in the optical properties due to the shift of the electron spectrum by the order of $100 \mathrm{meV}$. Calculations of fields of mechanical stress in $\mathrm{Ge} / \mathrm{Si}$ structures with quantum dots of $\mathrm{Ge}$ [14] showed that the most stressed area is located under the nanoisland basis, and the value of the silicon lattice deformation along the plane of the structure decreases with the distance to nanoisland. As a result, the band structure of silicon nanoislands surrounding is characterized by the presence of variations in the plane of the structure, so it is graded-band. The region of $\mathrm{Si}$ with the minimal value of band gap, which is also less than the band gap of the strained $\mathrm{Si}$, is located near the base nanoislands.

The decrease in the recombination rate of nonequilibrium electrons and holes photogenerated in the $\mathrm{Si}$ substrate is promoted by the electric field in the surface layer of depletion in the $p$-Si substrate. This field has a direction that facilitates the drift of non-equilibrium holes, which are photoexcited due to band-to-band transitions in $\mathrm{Si}$, from the illuminated surface. At the same time, non-equilibrium electrons fill the minimum of the potential energy near the surface. Spatial separation of non-equilibrium charge carriers, thus, reduces the probability of Shockley-Read recombination and delay PC kinetics at low temperatures. Fig. $2 b$ demonstrates the increase of $\tau$ with increasing the quantum energy, while the PC fall in the shortwave region of the photoconductivity spectrum at $h v>1.3 \mathrm{eV}$ was also observed (see Fig. 1). Usually, the decrease of the photoconductivity in this spectral range is explained by the fact that with increasing $h v$ more and more excitation radiation is absorbed in the surface region of $\mathrm{Si}$, where the recombination rate is higher in comparison with that in bulk of $\mathrm{Si}$. The observed changes in the shape of the spectra indicate that the rate of recombination of charge carrier photogenerated in the depletion layer of the $\mathrm{Si}$ substrate decreases during cooling when the spatial separation became more pronounced.

It should be noted that described long-term PC originates from spatial separation of electron-hole pairs photoexcited in Ge nanoislands and $\mathrm{Si}$ substrate. Let us analyze the peculiarities of long-term PC decay after excitation by photons that cannot excite free electronhole pairs in $\mathrm{Si}$. In this case, observed photoconductivity is monopolar: electrons photoexcited in Ge nanoislands are free to move. On the other hand, photoexcited holes are localized in the deep potential well of $\mathrm{Ge}$ nanoislands and can't contribute to in-plane transport. As a consequence, the Si regions under the base of the $\mathrm{Ge}$ nanoislands would have additional electrons as compared to the bulk $\mathrm{Si}$, while the Ge nanoislands can be considered as an efficient trap for holes. Under conditions of effective hole trapping by Ge nanoislands, recombination rate should be restricted by supply of minority carriers - electrons, which makes recombination more faster as compared with those after excitation of electron-hole pairs in the Si substrate.

\section{Conclusions}

In this study, PC measurements were used for detection of charge recombination in structures with $\mathrm{Ge} \mathrm{NCs}$ 
grown on $\mathrm{Si}$ (100) substrate. The in-plane photoconductivity, excited by light excitations with the photon energy below the band gap of $\mathrm{Si}$, is caused by interband transitions involving localized states of the valence band in the nanoislands. During investigation of the kinetics of photoconductivity in $\mathrm{Si} / \mathrm{Ge}$ nanoheterostructures, long-term relaxation of the photocurrent has been observed. Effects of the long-term photoconductivity decay in $\mathrm{Ge} / \mathrm{Si}$ heterostructures with Ge nanoislands has been found to be caused by variations of the electrostatic potential in the near-surface region of $\mathrm{Si}(100)$ substrate and spatial separation of electron-hole pairs between localized states of $\mathrm{Ge}$ nanoislands and states in the wetting layer and $\mathrm{Si}$. It has been found that, during selective photoexcitation of the $\mathrm{Ge}$ nanoislands, recombination of electron-hole pairs is defined by spatial separation of non-equilibrium charge carriers, when holes are trapped in the valence band states of $\mathrm{Ge}$ and electrons are in their silicon surroundings. The PC decay dependence on the excitation energy and temperature shows that $\mathrm{Ge}$ nanoislands are Shockley-Read recombination centers with a higher recombination rate as compared with $\mathrm{Si}$.

\section{Acknowledgements}

The work was carried out due to the support of the State Agency on Science, Innovations and Informatization of Ukraine (project number M/94-2014).

\section{References}

1. K. Bruner, Si/Ge nanostructures // Repts. Progr. Phys. 65, p. 27-72 (2002).

2. A.V. Dvurechenskii, A.I. Yakimov, N.P. Stepina, V.V. Kirienko, P.L. Novikov, SiGe nanodots in electro-optical SOI devices, in: Nanoscaled Semiconductor-on-Insulator Structures and Devices. Springer, 2007, p. 113-128.

3. G. Abstreiter, P. Schittenhelm, C. Engel, E. Silveira, A. Zrenner, D. Meertens, W. Jager, Growth and characterization of self-assembled Ge-rich islands on Si // Semicond. Sci Technol. 11, p. 1525 (1996).

4. A. Usami, N. Ujihara, T. Fujiwara, K. Sazaki, G. Nakajima, K. Shiraki, Enhanced quantum efficiency of solar cells with self-assembled $\mathrm{Ge}$ dots stacked in multilayer structure // Appl. Phys. Lett. 83, p. 1258 (2003).

5. N.T. Bagraev, A.D. Bouravleuv, L.E. Klyachkin, A.M. Malyarenko, S.A. Rykov, Self-ordered microcavities embedded in ultrashallow silicon $p-n$ junctions // Semiconductors, 34, p. 700-711 (2000).

6. J.M. Baribeau, X. Wu, N.L. Rowell, D.J. Lockwood, Ge dots and nanostructures grown epitaxially on $\mathrm{Si} / /$ Condensed Matter, 18, p. 139-174 (2006).

7. O.P. Pchelyakov, Yu.B. Bolkhovityanov, A.V. Dvurechenski, L.V. Sokolov, A.I. Nikiforov, A.I. Yakimov, B. Voigtlander, Silicon-germanium nanostructures with quantum dots: Formation mechanisms and electrical properties // Semiconductors, 34, p. 1229-1247 (2000).

8. M.K. Sheinkman and A.Ya. Shik, Long-term relaxation and residual conductivity in semiconductors // Fizika Tekhnika Poluprovodn. 10, p. 209-232 (1976), in Russian.

9. H.J. Queisser, D.E. Theodorou, Decay kinetics of persistent photoconductivity in semiconductors // Phys. Rev. B, 33, p. $4027-4033$ (1986).

10. H.J. Queisser, Nonexponential relaxation of conductance near semiconductor interfaces // Phys. Rev. Lett. 54, p. 234-236 (1985).

11. T.N. Sitenko, I.P. Tyagulskii, V.I. Lyashenko, V.S. Lysenko, Role of surface band bending in residual conductivity formation in epitaxial GaAs films // phys. status solidi (a), 30, p. $755-763$ (1975).

12. V. Kuryliuk, O. Korotchenkov, A. Cantarero, Carrier confinement in $\mathrm{Ge} / \mathrm{Si}$ quantum dots grown with an intermediate ultrathin oxide layer // Phys. Rev. B, 85, 075406-075416 (2012).

13. A.V. Dvurechenskii, A.I. Yakimov, Type-II Ge/Si quantum dots // Semiconductor Physics and Technology, 35, p. 1143-1153 (2001), in Russian.

14. O.A. Shegai, K.S. Zhuravlev, V.A. Markov, A.I. Nikiforov, O.P. Pchelyakov, Photoresistance of $\mathrm{Si} / \mathrm{Ge} / \mathrm{Si}$ structures with germanium quantum dots // Semiconductor Physics and Technology, 34, p. 1363-1367 (2000), in Russian.

15. A.A. Mykytiuk, S.V. Kondratenko, V.S. Lysenko, Yu.N. Kozyrev, Photocurrent spectroscopy of Ge nanoclusters grown on oxidized silicon surface // Proc. SPIE 9126, Nanophotonics, 9126, p. 3J (2014). 